\title{
Contactless Piston Position Transducer With Axial Excitation
}

\author{
Pavel Ripka, Andrey Chirtsov, and Vaclav Grim \\ Faculty of Electrical Engineering, Czech Technical University, 16627 Prague, Czech Republic
}

Existing piston position transducers require either drilling precise hole into the piston bar, or mounting permanent magnets or measuring device inside the pressurized cylinder. We present a new solution for aluminum pneumatic cylinders, which uses the ferromagnetic bar inside the solenoid as a marker and linear array of fluxgate sensors as a scale. Instead of relying on dc remanence we use active ac excitation; the reading is resistant against external fields, both dc and ac. Using sensor array allows to compensate for temperature effects. The linear stroke of the individual sensor is $40 \mathrm{~mm}$, so that array density should be about $30 \mathrm{~mm}$. $1 \mathrm{~mm}$ position resolution is achievable. The weak point of the new transducer is the response time: for fast moving pistons the excitation frequency should be high, which leads to weaker signal and lower resolution.

Index Terms-Magnetic sensors, piston position transducer, position measurement.

\section{INTRODUCTION}

$\mathbf{P}$ ISTON position transducers for the hydraulic and pneumatic cylinders are more demanded by industry, as they are necessary for fine control.

Position transducer for hydraulic cylinders is usually in the shape of a long probe which is inserted into the deep narrow blind hole in the cylinder rod [1]. Non-contact sensors based on the magnetostrictive principle (using toroidal permanent magnet in the piston) or variable inductance replace potentiometer sensors, which are cheap but have limited lifetime due to friction. The disadvantages of this type of sensors are the cost and reliability issues associated with the necessity of the long "gun drilled" hole in the rod and necessary fitting for the sensor, which resides inside the cylinder. Similar disadvantages exist for the microwave position sensors [2]. Vision-based sensors [3] and incremental optical piston position sensors [4], [5] were also developed, but they did not find industrial applications due to the reliability issues. Some systems use magnetic scale of a piston rod together with Hall sensors [6].

External monitoring of the hydraulic piston position is a challenge, as the walls of hydraulic cylinders are usually made of carbon steel which is ferromagnetic. The field of the permanent magnet embedded in the piston is therefore shielded by the ferromagnetic barrel wall and distorted by both the wall and rod. Precision better than $5 \mathrm{~mm}$ is therefore hardly achievable. Some special hydraulic cylinders such as those used in water hydraulic systems have composite shell. For these cylinders inductive displacement sensor can be built using a coil winding in the shell of the cylinder [7].

The pneumatic cylinders usually have aluminum wall which is transparent for the permanent magnet and therefore ideal for external transducers. Thanks to the simplicity and noncontact non-invasive capability these transducers are reliable and cost effective. The sensors being used for this application

Manuscript received March 9, 2017; revised April 27, 2017; accepted June 2, 2017. Date of publication June 13, 2017; date of current version October 24, 2017. Corresponding author: P. Ripka (e-mail: ripka@ @el.cvut.cz).

Color versions of one or more of the figures in this paper are available online at http://ieeexplore.iee.org.

Digital Object Identifier 10.1109/TMAG.2017.2715073 are mainly Hall and anisotropic magnetoresistor, rarely giant magnetoresitor.

However, permanent-magnet-based piston position transducers have several disadvantages as follows.

1) They are influenced by external magnetic fields including those induced by dc currents.

2) They require non-magnetic stainless steel piston rod, which is expensive. Aluminum cannot be used for this part because of the strength requirements.

3) Sensor cannot be mounted on existing cylinders if they are not equipped by the magnet. Usually the complete cylinder should be exchanged, which is difficult and expensive especially in the case of large machinery.

The distance between the permanent magnet and sensors is nonlinear function of the measured magnetic field. If the ferromagnetic objects are present in the close vicinity, the mentioned function is very complex. Nonlinear observer methods have been employed to accurately estimate the piston position in real time [8].

External dc magnetic sensors have been used also for the measurement of a piston position inside the cylinder of the free piston engine [9]. The disadvantage of such dc systems without the permanent magnet is that they rely on the remanence of ferromagnetic parts which may easily change with time and temperature.

In this paper, we introduce novel magnetic external position transducer for the pneumatic cylinders. It uses ac magnetic field excitation and detection by integrated fluxgate sensors.

\section{New AC Transducer}

Our new ac piston position transducer is using axial coil directly wound on the cylinder surface as a field source. The 2 or $3 \mathrm{~mm}$-thick electrically conducting cylinder wall has large attenuation, however, we show that at low frequencies the field inside the cylinder is still strong enough so that the cylinder movement can be observed by external fluxgate sensor.

\section{A. Transducer Design}

For the verification of this principle, we built a physical model of the pneumatic cylinder using $60 \mathrm{~mm}$ diameter barrel 
TABLE I

PARAMETERS OF THE EXCITATION COIL

\begin{tabular}{llll}
\hline \hline Coil length $(\mathrm{mm})$ & 480 & & \\
Number of turns & 808 & & \\
& $\mathrm{DC}$ & $100 \mathrm{~Hz}$ & $1 \mathrm{kHz}$ \\
$\mathrm{L}(\mathrm{mH})$ & - & 3,5 & 0,4 \\
$\mathrm{R}(\Omega)$ & 14.1 & 15 & 18 \\
\hline \hline
\end{tabular}

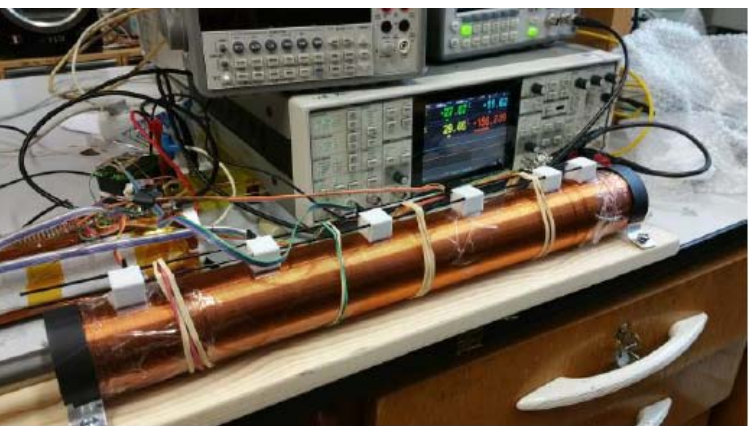

Fig. 1. Model of the pneumatic cylinder used for the experimental verification: the field source is the copper solenoid wound on the top of the aluminum cylindrical barrel. The sensors are inserted into the rectangular holders.

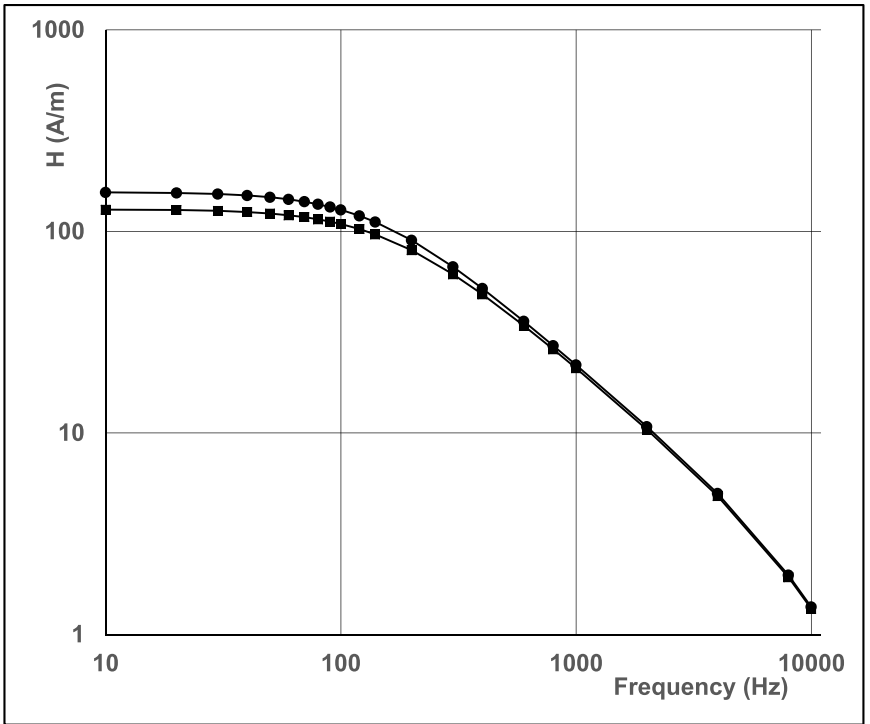

Fig. 2. Frequency dependence of the axial field in the middle of the cylinder $(\cdot)$ and at its ends (x).

pipe made of $2 \mathrm{~mm}$-thick aluminum, $10 \mathrm{~mm}$-thick aluminum piston, and $20 \mathrm{~mm}$ diameter steel piston rod. On the top of the cylinder, we wound single-layer axial coil with parameters in Table I. The model is shown in Fig. 1

The coil was supplied from the function generator with $50 \Omega$ internal resistance, so that the rms excitation current of $90 \mathrm{~mA}$ at low frequencies was decreasing with frequency to $70 \mathrm{~mA}$ at $100 \mathrm{~Hz}$. The maximum generated field in the center of the cylinder was $156 \mathrm{~A} / \mathrm{m}$ at $10 \mathrm{~Hz}$ and it was reduced mainly by the shielding effect of the aluminum cylinder to one half at $250 \mathrm{~Hz}$. The field at the end of the cylinder was $128 \mathrm{~A} / \mathrm{m}$ at the frequency of $10 \mathrm{~Hz}$. The frequency dependence

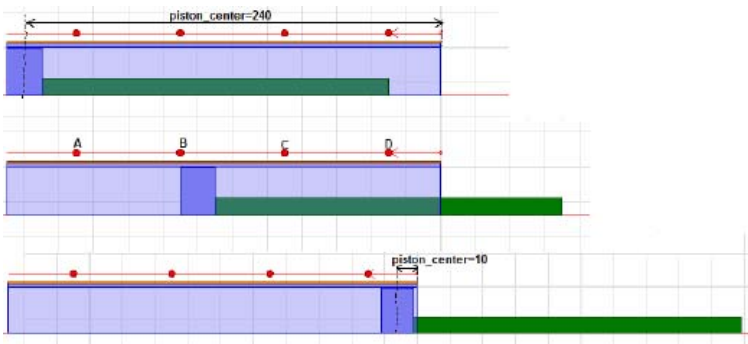

Fig. 3. Frequency dependence of the axial field in the middle of the cylinder $(\cdot)$ and at its ends $(\mathrm{x})$
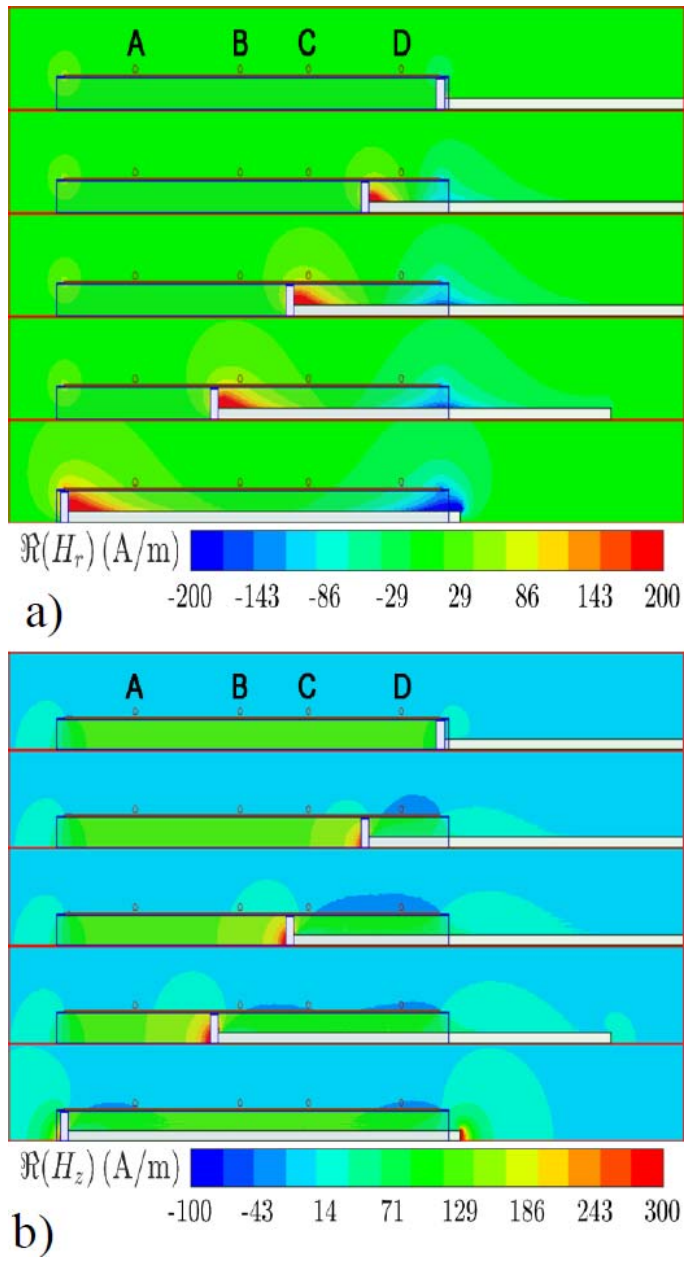

Fig. 4. FEM-simulated field for several positions of the piston. (a) Radial component. (b) Axial component. The excitation frequency was $2 \mathrm{~Hz}$. The location of the sensors is marked A to D.

of the internal field measured in the middle of the cylinder is shown in Fig. 2. The field at the end decreases to $50 \%$ at dc as theoretically predicted. This decrease is smaller for ac excitation as a consequence of the eddy currents; at $10 \mathrm{~Hz}$ the decrease is only $20 \%$.

\section{B. FEM Analysis}

In order to optimize the direction and position of the fluxgate sensors and also to find the optimum excitation frequency, we made extensive simulations based on finiteelement modelling (FEM) analysis. For the material properties, we have used the following values: 1) for the iron rod 

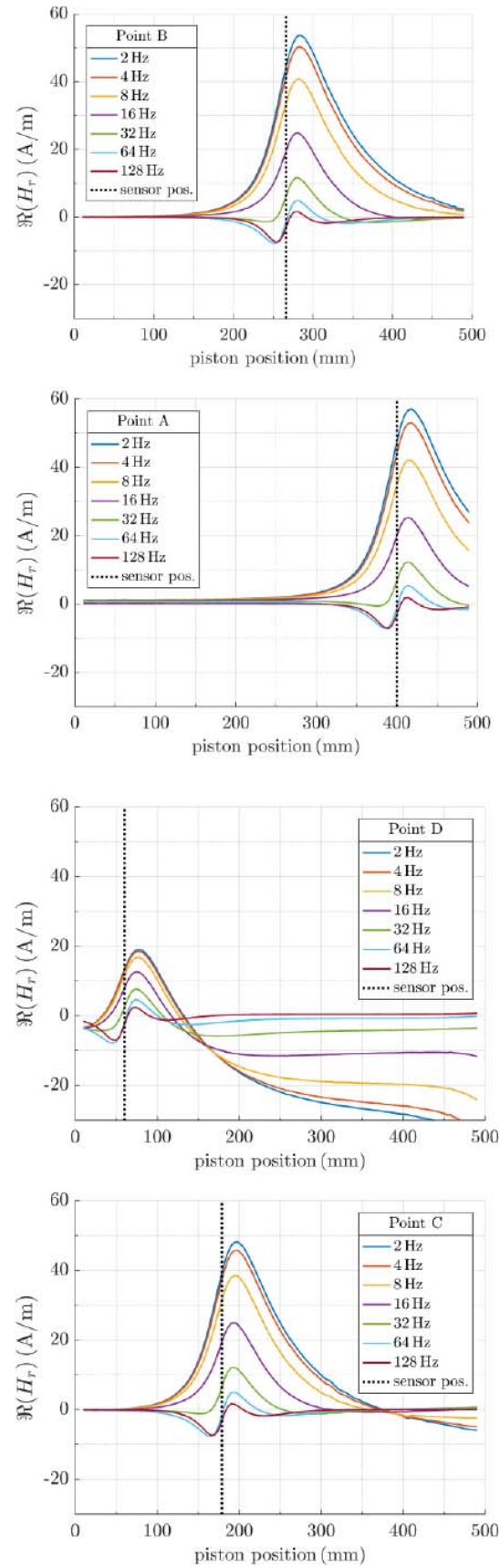

Fig. 5. Reading of the sensors in positions A, C, and D as the function of the piston position (FEM simulation).

relative permeability $\mu_{r}=50$ and conductivity $S=10$. $10^{6} \mathrm{~S} / \mathrm{m}$ and 2) for the aluminum cylinder and piston conductivity $S=38 \cdot 10^{6} \mathrm{~S} / \mathrm{m}$. The simulations were performed for numerous positions of the piston. Fig. 3 shows the definition of the piston position and location of the sensor.

Fig. 4 shows an example of the simulations: radial and axial field components calculated for four positions of the piston. The simulation shows that the field maximum is about $20 \mathrm{~mm}$ from the end of the bar and this distance is smaller at the limit position where the bar is completely out of the coil.

The simulated reading of the sensors in positions A to D as the function of the piston position is shown in Fig. 5 for the frequencies from 2 to $128 \mathrm{~Hz}$.
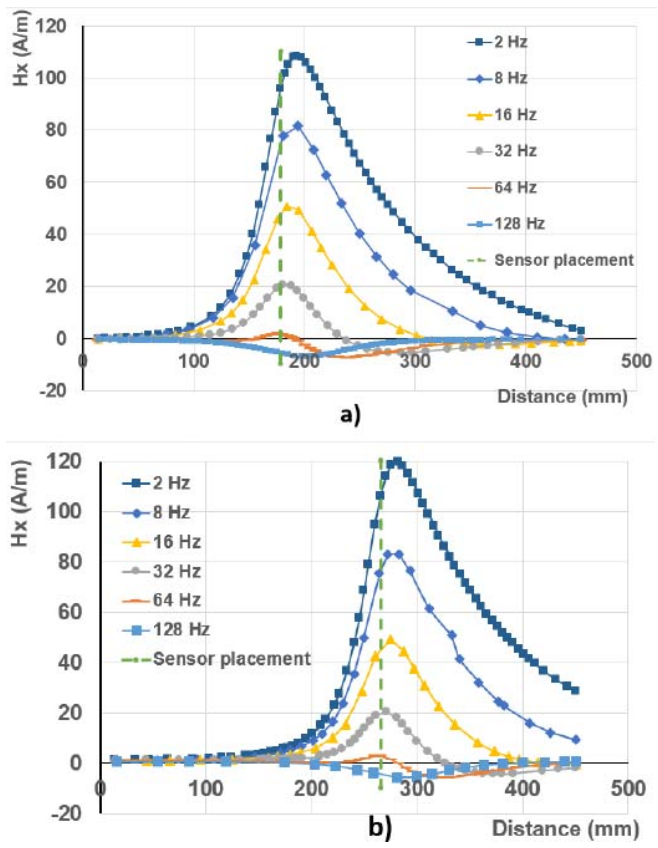

Fig. 6. Reading of the sensors in positions $\mathrm{B}$ and $\mathrm{C}$ as the function of the piston position (measured $X$ component).

The sensitivity decrease with frequency is caused by two effects as follows.

1) Eddy currents in the aluminum cylinder: the field from the excitation coil is attenuated by the shielding effect, as shown in Fig. 2, and the response from the rod is attenuated again before it reaches the sensors. These two shielding factors are not the same, as in the first case the attenuated field is in the axial direction, while in the second case it is in the radial direction. As an example, we can estimate that at $32 \mathrm{~Hz}$ the field of the source coil is attenuated only by $6 \%$, the overall drop of the sensitivity is $50 \%$.

2) Eddy currents in the piston bar. They are also the main source of phase shifts.

In order to separate the influence of the eddy currents in the aluminum piston, we ran the same set of simulations only for the iron rod without the piston. The simulations have shown that for the frequencies up to $100 \mathrm{~Hz}$, the contribution of the piston to the measured signal is negligible.

\section{Measurements}

The simulations were verified by the measurement. An array of integrated fluxgate magnetic sensors was mounted either in the radial direction (which is perpendicular to the primary field of the excitation coil) or in the axial position. This was possible only because the used sensor has low crossfield error [10]. We have used integrated fluxgates DRV425 manufactured by Texas Instruments [11]-[13]. The sensors were fixed in the plastic holders manufactured by $3-\mathrm{D}$ printing. The experimental stand is shown in Fig. 1. The piston position was monitored by resistive transducer with $0.1 \mathrm{~mm}$ accuracy. The output voltage of the fluxgate sensors was measured by SR865 DSP Lock-in amplifier. The reference signal was derived from the coil current. 


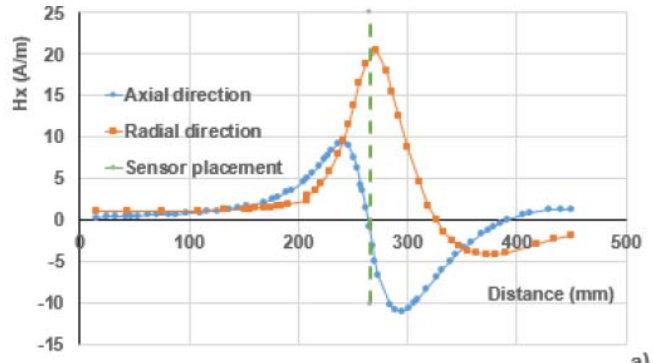

a)

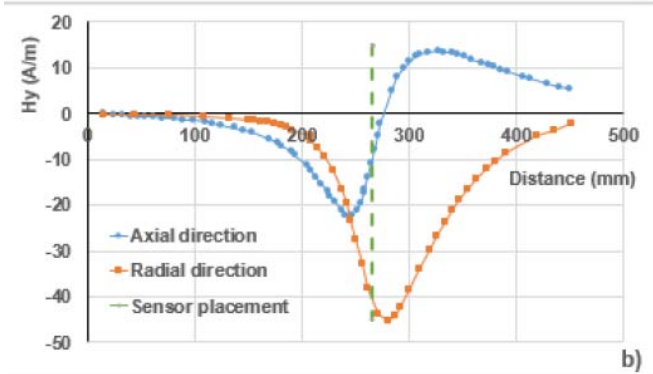

b)

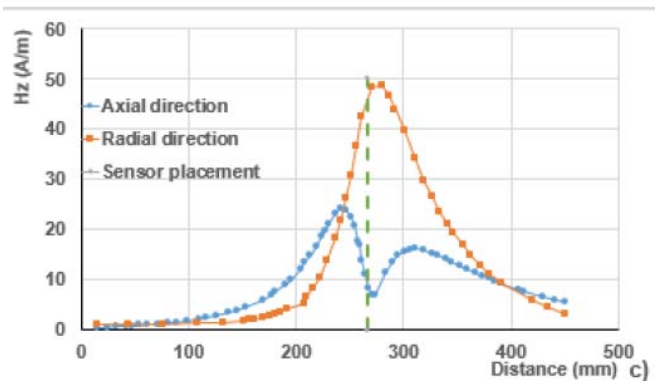

Fig. 7. Axial and radial field for $32 \mathrm{~Hz}$ excitation frequency. (a) $X$ component (in-phase with current). (b) $Y$ component. (c) Modulus.

In our case, we used DRV425EVM modules with $0.5 \mathrm{mT}$ range. However, using maximum range of $2 \mathrm{mT}$ would allow to increase the excitation current by the factor of 10 . In such case, the noise limit would drop below $0.1 \mathrm{~mm}$. The measured characteristics shown in Fig. 6 fit with the simulations. The sensitivity drops to one half at $20 \mathrm{~Hz}$ which is similar to the value predicted by the simulations.

Fig. 7 shows comparison of the field values in the axial and radial directions measured at the excitation frequency of $32 \mathrm{~Hz}$. We can find that the sensitivity in the radial direction is twice than the axial sensitivity. The axial field response is linear in the vicinity of the sensor location, which is a big advantage for the position sensing. The offset field from the source coil in the axial direction is only $1.6 \mathrm{~A} / \mathrm{m}$. At $32 \mathrm{~Hz}$, the phase shift is large which makes modulus measurement of the axial component useless. Sensor array should be used to cover strokes higher than $4 \mathrm{~cm}$.

\section{CONCLUSION}

Position of the piston in the pneumatic cylinder can be measured by the ac magnetic method. Using axial field excitation and an array of fluxgate sensors on the cylinder surface, an accuracy of $1 \mathrm{~mm}$ and the resolution of $0.1 \mathrm{~mm}$ is achievable. We have finally selected to measure the axial component of the field, which gives weaker signal compared to the radial direction, but gives linear range of $4 \mathrm{~cm}$. For longer strokes linear array of sensors spaced 2 to $3 \mathrm{~cm}$ should be used. The main advantages of the new method are as follows.

1) It can be used on existing cylinders, both the coil and sensors are mounted outside the cylinder.

2) No influence of external magnetic fields.

3) No need for expensive non-magnetic stainless steel piston rod.

4) Resistance to the rod geometrical and magnetic imperfections (verified by measurement with several rods, some of them with a curvature and some of them exposed to mechanical shocks).

The disadvantage of the axial transducer is that the excitation frequency should be kept low, which limits the dynamic response of the transducer.

\section{ACKNOWLEDGMENT}

The work of A. Chirtsov was supported by academic grant from Texas Instrument.

\section{REFERENCES}

[1] E. E. Herceg, "Taking a position on hydraulic cylinder sensors," Hydraulics Pneumatics, Tech. Rep., pp. 24-27, accessed on Jul. 03, 2017. [Online]. Avaliable: http://www.hydraulicspneumatics. com/cylinders-actuators/taking-position-hydraulic-cylinder-sensors

[2] S. Fericean, A. Hiller-Brod, A. D. Dorneich, and M. Fritton, "Microwave displacement sensor for hydraulic devices," IEEE Sensors J., vol. 13 no. 12, pp. 4682-4689, Dec. 2013.

[3] L. Shih-Yuan, L. Jyun, and S.-S. Lee, "The study of the piston driving and position sensing for a linearly moving piston pump," in Proc. CACS Int. Autom. Control Conf. (CACS), Kaohsiung, Taiwan, 2014, pp. 287-291.

[4] K. Suzumori, J. Tanaka, and T. Kanda, "Development of an intelligent pneumatic cylinder and its application to pneumatic servo mechanism," in Proc. IEEE/ASME Int. Conf. Adv. Intell. Mechatronics, Monterey, CA, USA, Jul. 2005, pp. 479-484.

[5] A. A. M. Faudzi, K. Suzumori, and S. Wakimoto, "Design and control of new intelligent pneumatic cylinder for intelligent chair tool application," in Proc. IEEE/ASME Int. Conf. Adv. Intell. Mechatronics, Singapore, Jul. 2009, pp. 1909-1914.

[6] S. Y. Yang, M. C. Lee, M. H. Lee, and S. Arimoto, "Measuring system for development of stroke-sensing cylinder for automatic excavator," IEEE Trans. Ind. Electron., vol. 45, no. 3, pp. 376-384, Jun. 1998.

[7] H. Sumali, E. P. Bystrom, and G. W. Krutz, "A displacement sensor for nonmetallic hydraulic cylinders," IEEE Sensors J., vol. 3, no. 6, pp. 818-826, Dec. 2003, doi: 10.1109/JSEN.2003.820333.

[8] Y. Wang, R. Madson, and R. Rajamani, "Nonlinear observer design for a magnetic position estimation technique," in Proc. 54th IEEE Conf. Decision Control (CDC), Osaka, Japan, Dec. 2015, pp. 6986-6991.

[9] S. Taghvaeeyan, R. Rajamani, and Z. Sun, "Non-intrusive piston position measurement system using magnetic field measurements," IEEE Sensors J., vol. 13, no. 8, pp. 3106-3114, Aug. 2013.

[10] P. Ripka, M. Janošek, M. Butta, S. W. Billingsley, and E. Wakefield, "Crossfield effect in commercial fluxgate and AMR sensors," J. Elect. Eng., vol. 61, no. 7/s, pp. 13-16, 2010.

[11] DRV425 Fluxgate Magnetic-Field Sensor, Texas Instruments Datasheet, accessed on Jul. 03, 2017. [Online]. Available: http://www.ti.com/product/DRV425/datasheet

[12] M. F. Snoeij, V. Schaffer, S. Udayashankar, and M. V. Ivanov, "Integrated fluxgate magnetometer for use in isolated current sensing," IEEE J. Solid-State Circuits, vol. 51, no. 7, pp. 1684-1694, Jul. 2016.

[13] D. W. Lee et al., "Fabrication and performance of integrated fluxgate for current sensing applications," in Proc. IEEE Trans. Magn., to be published, doi: 10.1109/TMAG.2017.2713244. 